\title{
Glukokortikoide - Wann ist der Einsatz gerechtfertigt?
}

\author{
Hermann Ammer
}

Glukokortikoide zählen in der Kleintierpraxis zu den am häufigsten eingesetzten Arzneimitteln. Sie sind unverzichtbar bei der Behandlung entzündlicher und immunmediierter Erkrankungen. Sie werden aber auch häufig leichtfertig bei einer Vielzahl weiterer Indikationen eingesetzt, bei denen das Glukokortikoid die Grunderkrankung maskieren oder verschlechtern kann. Zudem ist bei einigen Indikationen der therapeutische Nutzen nicht eindeutig geklärt. Der folgende Artikel fasst die grundsätzlichen Anwendungsrichtlinien für Glukokortikoide zusammen und diskutiert einige umstrittene Einsatzgebiete aus Sicht der klinischen Pharmakologie.

\section{Einleitung}

Kortisol ist ein systemisch wirksames Steroidhormon, das in der Zona fasciculata der Nebenniere gebildet wird. Seine biologische Bedeutung liegt in der Aufrechterhaltung der Homöostase bei Hunger- und Stresszuständen sowie in der Regulation von Reparaturvorgängen nach Gewebeverletzungen. Die Synthese und Freisetzung von Kortisol wird durch einen negativen Feedback-Mechanismus unter Beteiligung hypothalamischer und hypophysärer Hormone reguliert. Dieser führt beim Hund zu mehreren, über den Tag verteilten Freisetzungspeaks. In Stresssituationen kann die Synthese von Kortisol kurzzeitig um das 10fache gesteigert werden. Es ist daher Konsens, im Rahmen einer Substitutionstherapie der Nebennierenrinden-Insuffizienz die Tagesdosis auf mehrere Einzelgaben pro Tag aufzuteilen und in Belastungssituationen zu erhöhen [1].

\section{Klinische Pharmakologie Pharmakokinetische Eigenschaften}

Die synthetischen Glukokortikoide leiten sich von Kortisol ab. In der Kleintierpraxis wird hauptsächlich Prednisolon verwendet, das als Standardglukokortikoid gilt. Seine volle Wirkung tritt bei einer Dosis von 1-1,5 mg/kg ein, bei der bereits sämtliche Rezeptoren besetzt sind. Durch Veränderungen am Steroidgerüst des Prednisolons wurden weitere Vertreter gewonnen, die sich durch eine gesteigerte glukokortikoide und abgeschwächte mineralokortikoide Wirkung auszeichnen. Ihr Wirkprofil bleibt dagegen unverändert. Aufgrund der höheren Wirkpotenz nimmt die Wirkdauer bei vergleichbarer Dosierung lediglich zu.

Als Besonderheit bei den Glukokortikoiden ist zu beachten, dass ihre pharmakologischen Eigenschaften auch durch die Darreichungsform bestimmt werden. Als freier Alkohol sind Glukokortikoide in Wasser beinahe unlöslich. Sie werden daher enteral gut resorbiert und eignen sich für die orale Applikation.

Für Injektionslösungen müssen Glukokortikoide dagegen verestert werden. Bei wässrigen Injektionslösungen (Dihydrogenphosphat, Hydrogensuccinat etc.) liegen die Wirkstoffe in freier Form vor, sodass die Wirkung schnell eintritt. Bei Suspensionen (Acetat, Acetonid, Dipropionat etc.) liegen die Wirkstoffe dagegen nicht gelöst vor, woraus ein Depoteffekt mit verzögerter Wirkstofffreisetzung resultiert. Bei den Dosierungsempfehlungen und den Angaben für die Wirkdauer von Glukokortikoiden ist daher immer die vorliegende Arzneiform zu berücksichtigen. Die Literaturangaben beziehen sich in der Regel auf die schnell wirkenden Präparate.
Die Eigenschaften der derzeit in der Kleintiermedizin eingesetzten Glukokortikoide werden in $>$ Tab. 1 zusammengestellt $[1,14]$.

\section{Indikationen}

Glukokortikoide verfügen über ein breites Wirkspektrum, das durch ihre komplexen zellulären Wirkmechanismen erklärt werden kann. Auf zellulärer Ebene wirken Glukokortikoide zunächst als Genregulatoren, die ihre Wirkungen über die Aktivierung zytosolischer Rezeptoren vermitteln. Welche Gene am Ende reguliert werden und wie lange die biologische Wirkung anhält, hängt maßgeblich von der Halbwertszeit der regulierten Proteine, vom Zelltyp, vom Wirkstoff und von der verabreichten Dosis ab. Darüber hinaus besitzen Glukokortikoide eine membranstabilisierende Wirkung, indem sie sich aufgrund ihrer strukturellen Ableitung vom Cholesterin direkt in die Zellmembran einlagern. Zusammengenommen resultieren die genannten Mechanismen in der antiphlogistischen, antiproliferativen und immunsuppressiven Wirkung der Glukokortikoide. Daraus lassen sich die verschiedenen Indikationsgebiete ableiten $($ Kasten S. 14).

\section{konkret}

Jeder Einsatz von Glukokortikoiden erfordert aufgrund der unerwünschten Nebenwirkungen eine strenge Indikationsstellung und eine gründliche Nutzen-Risiko-Abwägung.

\section{Unerwünschte Nebenwirkungen}

Für den klinischen Einsatz der Glukokortikoide ist von Bedeutung, dass ihre Wirkung bis auf den direkten membranstabilisierenden Effekt erst mit einer zeitlichen Verzögerung von einigen 
Stunden bis Tagen eintritt. Zudem können bei jeder Anwendung inhärente, also kalkulierbare unerwünschte Arzneimittelwirkungen auftreten, die sich in Abhängigkeit der Wirkstoffstärke, der Dosierung und der Anwendungsdauer entwickeln. Mit steigenden Dosierungen werden zunächst die metabolischen Ef- fekte verstärkt, bevor es zu einer Hemmung der Nebennierenrinden-Hypothalamus-Hypophysen-Achse und zur Ausbildung des Cushing-Syndroms kommt. Hunde entwickeln dabei zunächst eine Polyphagie und Polydipsie, während Katzen mit Hyperglykämie reagieren. Bei längerfristiger Anwendung höherer Do- sierungen treten beim Hund zudem Wundheilungsstörungen, Hautatrophie und -kalzinose auf. Ebenfalls steigt das Infektionsrisiko an. Bei der Katze werden nach Langzeitanwendung Polyurie, Polyphagie und Gewichtszunahme beobachtet $[1,14]$.

Tab. 1 Pharmakologische Eigenschaften gebräuchlicher Glukokortikoide.

\begin{tabular}{|c|c|c|c|c|}
\hline $\begin{array}{l}\text { Wirk- } \\
\text { dauer }\end{array}$ & Wirkstoff & veterinärmedizinische Präparate & Dosierung Hund (mg/kg) & $\begin{array}{l}\text { relative } \\
\text { mineralokorti- } \\
\text { koide Wirkung } \\
(\text { Kortisol }=1)\end{array}$ \\
\hline$<12 \mathrm{~h}$ & $\begin{array}{l}\text { Hydrokortison } \\
\text { (Kortison) }\end{array}$ & $\begin{array}{l}\text { Injektionspräparate: keine } \\
\text { orale Applikation: keine } \\
\text { lokale Applikation: } \\
\text { als Cortavance }{ }^{\circledR} 0,584 \mathrm{mg} / \mathrm{ml} \\
\text { (als Hydrokortisonaceponat; } \\
\text { Wirkdauer > } 12 \text { h) }\end{array}$ & $\begin{array}{l}\text { Substitutionstherapie: } \\
1-2 \mathrm{mg} / \mathrm{kg} \text { p.o., } 2 \text { × tgl. } \\
\text { Allergien: } \\
5 \mathrm{mg} / \mathrm{kg} \text { p.o., auf } 2-3 \text { Tagesdosen } \\
\text { Anaphylaxie und Endotoxinschock: } \\
\text { bis } 50 \mathrm{mg} / \mathrm{kg} \text { langsam i.v., alle } 2-3 \mathrm{~h} \\
\text { Atopische Dermatitis: } \\
1,52 \mathrm{\mu g} \text { Hydrocortisonaceponat } / \mathrm{cm}^{2} \text {, } \\
1 \times \text { tgl. für } 7 \text { Tage }\end{array}$ & 1 \\
\hline $12-36 \mathrm{~h}$ & Prednisolon & $\begin{array}{l}\text { Depotpräparate zur Injektion: } \\
\text { Prednisolon ad us. vet. } 10 \mathrm{mg} / \mathrm{ml} \text {, } \\
\text { Prednisolonacetat } 1 \% \\
\text { orale Applikation: } \\
\text { Prednisolon } 5 \mathrm{mg} \text {, Prednisolon } 50 \mathrm{mg}\end{array}$ & $\begin{array}{l}\text { Substitutionstherapie: } \\
\text { 0,25-0,5 mg/kg p.o., } 1 \times \text { tgl. } \\
\text { - Anaphylaxie und Endotoxinschock: } \\
10-30 \text { mg/kg i.v., alle } 8-12 \text { h } \\
\text { - Initialbehandlung bei allergischen oder } \\
\text { entzündlichen Erkrankungen: } \\
\text { 1-3 mg/kg i.m., p.o., auf 2-3 Tages- } \\
\text { dosen } \\
\text { Erhaltungsdosis bei allergischen oder } \\
\text { entzündlichen Erkrankungen: } \\
\text { ca. } 0,5-1 \text { mg/kg p.o., } 1 \times \text { tgl. oder } \\
\text { alternierend jeden } 2 \text {. Tag }\end{array}$ & 0,3 \\
\hline $12-36 \mathrm{~h}$ & $\begin{array}{l}\text { Methyl- } \\
\text { prednisolon }\end{array}$ & $\begin{array}{l}\text { schnell wirksame Injektionspräparate: } \\
\text { Medrate Solubile ad us. vet. } 125 / 500 \text { mg } \\
\text { Depotpräparate zur Injektion: } \\
\text { Depo-Medrate }{ }^{\circledR} \text { ad us. vet. } 40 \text { mg/ml }\end{array}$ & $\begin{array}{l}\text { Anaphylaxie und Endotoxinschock: } \\
4-10 \mathrm{mg} / \mathrm{kg} \text { i.v., alle 3-6 h }\end{array}$ & 0 \\
\hline $12-48 \mathrm{~h}$ & Triamcinolon & $\begin{array}{l}\text { lokale Applikation: } \\
\text { Panolog }{ }^{\circledR}\end{array}$ & $\begin{array}{l}\text { Entzündungshemmung: } \\
0,2-0,3 \mathrm{mg} / \mathrm{kg} \text { i.m. eines umgewidmeten } \\
\text { Depotpräparats, } 1 \text {-malig }\end{array}$ & 0 \\
\hline$>48 \mathrm{~h}$ & Flumetason & - & $\begin{array}{l}\text { Entzündungshemmung: } \\
0,01-0,02 \mathrm{mg} / \mathrm{kg} \text { i.v., i.m., 1-malig, kann } \\
\text { in Ausnahmefällen wiederholt werden }\end{array}$ & 0 \\
\hline$>48 \mathrm{~h}$ & Dexamethason & $\begin{array}{l}\text { schnell wirksame Injektionslösungen: } \\
\text { Dexa-ject } 2 \mathrm{mg} / \mathrm{ml} \text {, Dexamethason } \\
\text { ad us. vet. } 2 \mathrm{mg} / \mathrm{ml} \text {, Dexasel } 2 \mathrm{mg} / \\
\text { ml, Dexatat } 2 \mathrm{mg} / \mathrm{ml} \text {, Hexadreson }{ }^{\circledR} \\
2 \mathrm{mg} / \mathrm{ml} \text {, Rapidexon Albrecht } 2 \mathrm{mg} / \mathrm{ml} \text {, } \\
\text { Dexamethason } 4 \mathrm{mg} / \mathrm{ml} \\
\text { - Depotpräparate zur Injektion: } \\
\text { Dexadreson }{ }^{\circledR} \text { forte } 1,32 / 2,67 \mathrm{mg} / \mathrm{ml} \text {, } \\
\text { Voren Suspension } 1 \mathrm{mg} / \mathrm{ml} \\
\text { orale Applikation: } \\
\text { Dexamethason 0,5 mg } \\
\text { lokale Applikation: } \\
\text { Dexamethason in DMSO, Hydrocortisel, } \\
\text { Prurivet S }\end{array}$ & $\begin{array}{l}\text { Anaphylaxie und Endotoxinschock: } \\
2-5 \mathrm{mg} / \mathrm{kg} \text { i.v., bei Bedarf nach 8-12 h } \\
\text { wiederholen } \\
\text { allergische und entzündliche Erkran- } \\
\text { kungen: } \\
0,05 \mathrm{mg} / \mathrm{kg} \text { p.o., } 1 \times \text { tgl. oder } \\
0,1-0,25 \mathrm{mg} \text { i.v., i.m., } 1 \times \text { tgl. oder } \\
\text { alternierend jeden } 2 \text {. Tag }\end{array}$ & 0 \\
\hline$>48 \mathrm{~h}$ & Betamethason & $\begin{array}{l}\text { systemische Applikation: } \\
\text { Celestovet }{ }^{\circledR} 12,0 / 3,948 \mathrm{mg} / \mathrm{ml} \\
\text { - Iokale Applikation: } \\
\text { Isaderm }^{\circledR} \text { Gel, Osurnia }\end{array}$ & $\begin{array}{l}\text { bei entzündlichen Erkrankungen des } \\
\text { Bewegungsapparats: } \\
0,18-0,35 \mathrm{mg} / \mathrm{kg} \text { i.m., i.a., } 1 \text {-malig, } \\
\text { i.m.-Injektion kann bei Bedarf bis zu } 4 \text { × } \\
\text { wiederholt werden }\end{array}$ & 0 \\
\hline
\end{tabular}




\section{Indikationsgebiete für Glukokortikoide}

\section{Nebenniereninsuffizienz}

Im Rahmen der Substitutionstherapie werden die physiologischen Wirkungen der Glukokortikoide auf den Protein-, Glukose- und Fettstoffwechsel ausgenutzt. Hierfür werden in der Regel die natürlich vorkommenden Glukokortikoide in niedriger Dosierung verwendet.

\section{Entzündungen}

Um pharmakologische Wirkungen zu erzielen, werden höhere Dosierungen oder stärker wirksame Glukokortikoide benötigt. In diesen sog. „supraphysiologischen Dosierungen“ besitzen Glukokortikoide u. a. eine ausgeprägte antiinflammatorische Wirkung.

Daran sind die folgenden Mechanismen beteiligt:

- Abschaltung proinflammatorischer Gene (NFkB)

- Blockade der Arachidonsäurekaskade auf Ebene der Phospholipase A2

- Hemmung mesenchymaler Prozesse

- Beeinflussung zellulärer Entzündungsreaktionen

- direkte Membranstabilisierung

Hieraus resultiert bei allen akuten Entzündungen ein antiexudativer und analgetischer Effekt, der eine Chronifizierung der Prozesse durch Hemmung der bindegewebigen Reaktion und leukozytäre Infiltration verhindert.

\section{Allergien und Autoimmunerkrankungen}

In höheren Dosierungen besitzen Glukokortikoide neben ihrer antiinflammatorischen Wirkung ebenfalls antiallergische und immunsuppressive Wirkungen. Diese beruhen auf einer ausgeprägten antiproliferativen Wirkung und einer Hemmung der Mediatorenfreisetzung, der Makrophagenfunktion und der T-Zell-vermittelten Effekte. Diese Effekte können therapeutisch bei akuten Allergien, beim Endotoxinschock und bei Autoimmunkrankheiten ausgenutzt werden.

\section{Tumorerkrankungen}

Aufgrund ihrer antiproliferativen und lympholytischen Wirkung können Glukokortikoide auch alleine oder in Kombination mit Zytostatika u. a. zur Behandlung lymphatischer Tumore bei Hund und Katze eingesetzt werden.

\section{Therapiegrundsätze}

Aus dem Wirkmechanismus und der Gefahr für unerwünschte Wirkungen können folgende allgemein bekannte Anwendungsrichtlinien abgeleitet werden ( Kasten S. 16).

\section{Gegenanzeigen}

Als allgemeine Kontraindikationen gelten $[1,14]$ :

- bakterielle Infektionen

- Systemmykosen

- Diabetes mellitus

- Lebererkrankungen

- ein erhöhtes Risiko für Magen-DarmUlzera

Kontrovers diskutiert wird in der Tiermedizin nach wie vor die kombinierte Anwendung von Glukokortikoiden mit nicht steroidalen Antiphlogistika
(NSAID). Hintergrund ist, dass NSAID und Glukokortikoide sequenziell in den Arachidonsäurestoffwechsel eingreifen und es dadurch zu einer synergistischen Hemmung der Prostaglandinsynthese kommt. Therapeutisch kommt dieser Effekt jedoch nur eingeschränkt zum Tragen, da der Wirkeintritt der Glukokortikoide im Gegensatz zu den NSAID zeitlich verzögert ist.

\section{Das Risiko für gastrointestinale Neben- wirkungen steigt bei einer klinisch relevanten Wirkverstärkung daher unverhältnismäßig stark an.}

So wurden nach einer 30-tägigen Behandlung von Hunden mit Prednisolon $(0,5 \mathrm{mg} / \mathrm{kg})$ und einer um $50 \%$ reduzierten Dosis von Ketoprofen $(0,25 \mathrm{mg} / \mathrm{kg})$ oder Meloxicam $(0,1 \mathrm{mg} / \mathrm{kg})$ signifikant häufiger gastrointestinale Läsionen, schwere Beeinträchtigungen der Nierenfunktion und -integrität sowie der Plättchenaggregation nachgewiesen [11].

Die klinische Evidenz für eine erfolgreiche Prävention gastrointestinaler Komplikationen bei gleichzeitiger Verabreichung gastroprotektiver Medikationen wie Misoprostol, Hemmstoffe der Protonenpumpe oder H2-Antihistaminika ist gering [7]. Die Kombination von Glukokortikoiden mit einem NSAID wird daher heute nur noch in Sonderfällen akzeptiert, z.B. bei lokaler Applikation eines Glukokortikoids und gleichzeitiger systemischer Verabreichung eines NSAID.

\section{Evidenzbasierte Medizin}

Glukokortikoide eignen sich grundsätzlich für die Behandlung entzündlicher, allergischer und immunologischer Erkrankungen. Darüber hinaus werden sie jedoch auch bei einer Reihe weiterer Indikationen eingesetzt, bei denen ihr therapeutischer Nutzen nicht in jedem Fall ausreichend gesichert ist. Ein Grund hierfür ist, dass Glukokortikoide nur symptomatisch wirken und bei jeder Anwendung vielfältige Auswirkungen auf die Homöostase und den Heilungsverlauf auftreten können.

Es stellt sich daher die Frage, bei welchen der genannten Anwendungsgebieten der Einsatz von Glukokortikoiden für den Patienten von Vorteil ist? Eine Antwort hierfür versucht die evidenzbasierte Medizin zu geben, die aufgrund einer systematischen Auswertung aller verfügbaren wissenschaftlichen Informationen eine für den individuellen Patienten bestmög-

\section{Wissenswert}

Bei folgenden Erkrankungen ist der Einsatz von Glukokortikoiden differenziert zu betrachten:

- Kreislaufschock

- Erkrankungen des Bewegungsapparats

- ZNS-Erkrankungen

- Erkrankungen des Magen-DarmTrakts

- Hauterkrankungen 


\section{Anwendungsrichtlinien für Glukokortikoide}

- Glukokortikoide wirken symptomatisch und können die Grunderkrankung maskieren. Daher sind vor jeder Indikationsstellung die diagnostischen Maßnahmen abzuschließen.

- Bei jeder Anwendung ist ein Therapieziel zu definieren, an dem die Wirkung beurteilt und die Dosierung angepasst werden kann.

- Bei akuten Indikationen können einmalig höhere Dosen eines gelösten Glukokortikoids verabreicht werden.

- Für eine Langzeittherapie wird meist Prednisolon oral verabreicht. Um die Gefahr für Nebenwirkungen zu reduzieren, wird die Dosis nach dem Eintreten der erwünschten klinischen Wirkung schrittweise im Abstand von 1 Woche bis zum Erreichen der niedrigsten, gerade noch wirksamen Erhaltungsdosis reduziert.

- Je nach verwendetem Glukokortikoid kann es bereits wenige Tage nach dem Absetzen des Präparats zur Ausbildung von Entzugserscheinungen (Addison-Krise) kommen. Nach einer Anwendungsdauer von mehr als 2 Wochen soll die Therapie ausgeschlichen werden, indem abnehmende Dosen nur noch jeden 2. oder 3. Tag verabreicht werden. Nach Langzeitanwendung über 1 Monat ist die ausschleichende Therapie über 2-3 Wochen durchzuführen.

- Für ausgewählte Indikationsgebiete stehen Wirkstoffe mit besonderen pharmakokinetischen Eigenschaften wie Budesonid (Enteropathie) und Fludrocortison (inhalativ bei Asthma) zur Verfügung.

liche Therapieempfehlung ausarbeitet [4]. So gilt es heute als anerkannt, dass Glukokortikoide nicht mehr bei allen Formen des Kreislaufschocks sinnvoll sind. Sie zeigen lediglich beim anaphylaktischen Schock und beim Endotoxinschock eine ausreichende Wirksamkeit. Beim hypovolämischen Schock werden sie heute nicht mehr empfohlen. Bei Erkrankungen des Bewegungsapparats bleibt ihre Anwendung ebenfalls nur auf nicht infektiöse entzündliche Veränderungen beschränkt. Bei degenerativen Erkrankungen wie der Osteoarthrose sollen Glukokortikoide dagegen aufgrund ihrer knorpelkatabolen Eigenschaften nicht mehr angewendet werden [1]. Weniger eindeutig ist die Einschätzung ihrer Wirksamkeit bei Erkrankungen des ZNS, des Magen-Darm-Trakts und der Haut. Im Folgenden soll daher der aktuelle Diskussionsstand zum Einsatz von Glukokortikoiden bei diesen Indikationen wiedergegeben werden.

\section{ZNS-Erkrankungen}

Durch die Stabilisierung der mikrovaskulären Integrität werden Glukokortikoide häufig bei folgenden Erkrankungen eingesetzt:

- akute Wirbelsäulenverletzungen

- Traumata

- Diskopathie
- ZNS-Traumata

- nicht infektiöse Meningoenzephalitis

- septische Meningitiden

- Hydrozephalus

- nekrotisierende Vaskulitiden

- Spondylopathien

- Neoplasien

Aufgrund der teilweise komplexen Krankheitsgeschehen in Verbindung mit einer relativ dünnen Datenlage in der Kleintiermedizin ist die Beurteilung des therapeutischen Nutzens von Glukokortikoiden nur bei einigen Indikationen möglich, die im Folgenden besprochen werden.

\section{Akute Wirbelsäulenverletzungen}

Hierzu zählen Traumata und vor allem die Diskopathie des Hundes. Die Prognose bei diesen Erkrankungen wird maßgeblich durch die Lokalisation des Traumas und den Zeitpunkt der Vorstellung des Patienten bestimmt. Die spezifische Therapie mit Glukokortikoiden zielt auf eine Prävention von Sekundärschäden wie Thrombosen und Nekrosen ab, die sich als Reaktion auf die Freisetzung von Entzündungsmediatoren, Neurotransmittern und reaktiven Sauerstoffspezies hin entwickeln. In diesem Zusammenhang konnte nur für hochdosiertes Methylprednisolon eine antioxidative Wir- kung nachgewiesen werden, nicht aber für Prednisolon oder Dexamethason. Im geschädigten Gewebe konnte keines der Glukokortikoide die Bildung von Prostaglandinen hemmen.

Untersuchungen in der Humanmedizin konnten bei Patienten verbesserte motorische und sensorische Leistungen beobachten, die innerhalb von 8 Stunden nach dem Ereignis mit Methylprednisolon behandelt wurden. Eine spätere Verabreichung war ohne Einfluss auf die genannten Endpunkte, erhöhte aber das Risiko für die Entstehung einer Sepsis und Pneumonie signifikant. Als weitere unerwünschte Arzneimittelwirkungen wurden gastrointestinale Blutungen, Wundinfektionen und Hyperglykämie beobachtet [13]. Für Hund und Katze liegen aktuell keine aussagekräftigen Studien vor, die einen therapeutischen Vorteil für Methylprednisolon und andere Glukokortikoide bei der Diskopathie belegen. Eine routinemäßige Anwendung von Glukokortikoiden bei der Diskopathie kann daher gegenwärtig nicht empfohlen werden [6].

\section{ZNS-Trauma}

In der Humanmedizin hat sich bei der Behandlung des akuten Schädel-Hirn-Traumas mit Glukokortikoiden im letzten Jahrzehnt ein Paradigmenwechsel vollzogen. Galt früher ein drohendes Gehirnödem als absolute Indikation für Glukokortikoide, förderte eine systematische Auswertung mehrerer randomisierter prospektiver Studien eine erhöhte Mortalität 14 Tage und 6 Monate nach der Verabreichung von Glukokortikoiden zu Tage [1].

Akute Schädel-Hirn-Traumata weisen auch bei Hund und Katze eine hohe Morbidität und Mortalität infolge der komplexen intra- und extrakraniellen Regulationsmechanismen auf [15]. Die Pharmakotherapie zielt daher auch bei dieser Indikation auf eine Minimierung der sekundären Gewebeschädigung ab. Neben systemischen Maßnahmen zur Aufrechterhaltung der Gewebeperfusion, der Atmung und Blutversorgung werden spezifische Maßnahmen zur Reduktion des intrakranialen Druckes mit Osmodiuretika und zur Unterdrückung von Krampfanfällen mit Benzodiazepinen durchge- 
führt. Nachdem eigene Studien für die Tiermedizin aber fehlen, werden Glukokortikoide heute entsprechend der Erfahrungen in der Humanmedizin nicht mehr für die Behandlung des SchädelHirn-Traumas bei Hund und Katze empfohlen $[6,15]$.

\section{Nicht infektiöse Meningoenzephalitis}

Die Beurteilung des Therapieerfolgs bei der Behandlung der nicht infektiösen Meningoenzephalitis beim Hund wird dadurch erschwert, dass die Erkrankung zu Behandlungsbeginn nicht sicher diagnostiziert werden kann. Die Studienkollektive sind daher sehr heterogen. In einer Übersichtsarbeit aus dem Jahr 2010 wurden insgesamt 8 retrospektive Studien zur Behandlung der granulomatösen Meningoenzephalitis und anderer Enzephalitiden unbekannten Ursprungs beim Hund systematisch ausgewertet und die Wirksamkeit von Glukokortikoiden im Vergleich zu anderen Therapieoptionen betrachtet. Als Endpunkt für die Beurteilung der Wirksamkeit wurde die Überlebenszeit der Tiere herangezogen [9]. Je nach Studie konnte für Prednisolon in einer Dosierung von 0,25-2 mg/ $\mathrm{kg} 2 \times \mathrm{tgl}$. eine mittlere Überlebenszeit zwischen 28-357 Tagen $(n=43)$ bestimmt werden. Bei der Kombination von Prednisolon mit einem Immunsuppressivum verlängerte sich die Überlebenszeit auf 240-590 Tage $(n=96)$. Die einzelnen Studien unterschieden sich jedoch in Bezug auf die getroffenen Einschlusskriterien und die verwendeten Dosierungen der Glukokortikoide und Immunsuppressiva. Aus diesem Grund werden weitere prospektive Studien gefordert, um eine fundierte Empfehlung für ein Dosierungsregime mit Glukokortikoiden auszusprechen.

\section{Chronisch-entzündliche Darm- erkrankung des Hundes}

Bei der „Chronic Inflammatory Bowel Disease“ (CIBD) handelt es sich um eine gesteigerte Immunreaktion der Darmschleimhaut gegen intestinale Mikroben, die zusammen mit diätetischen Maßnahmen grundsätzlich mit Glukokortikoiden behandelt werden kann.

\section{Wesentlich für die Indikationsstellung ist, dass vorab andere gastrointestinale Erkrankungen wie eine Nahrungs- mittelallergie und infektiöse Ursachen sicher ausgeschlossen werden.}

Bei diesen Fällen wäre der Einsatz von Glukokortikoiden nicht gerechtfertigt.

Für die Beurteilung verschiedener Therapieoptionen der chronisch-entzündlichen Darmerkrankung liegen erste Studien zur klinischen Wirksamkeit von Prednisolon und Budesonid vor. Budesonid wurde für diese Indikation entwickelt, da es einen ausgeprägten „FirstPass-Effekt“ in der Leber aufweist und somit nur lokal in der Darmschleimhaut wirken soll, unter Umgehung systemischer Nebenwirkungen. In einer Studie mit 54 Hunden, die an unterschiedlichen Schweregraden der chronisch-entzündlichen Darmerkrankung litten, konnte bei einer Monotherapie mit Prednisolon in einer Dosis von $1 \mathrm{mg} / \mathrm{kg}$ bei $80 \%$ der Tiere eine Remission erzielt werden. Als Endpunkte wurden der „Chronic Inflam- matory Bowel Disease“(CIBD)-ActivityIndex oder laborchemisch das C-reaktive Protein (CRP) herangezogen. Die zusätzliche Gabe von Metronidazol hatte keinen Effekt auf die Remissionsrate [10].

Die Wirksamkeit von Budesonid wurde in einer randomisierten, doppelt verblindeten prospektiven Studie an 40 neu an chronisch-entzündlicher Darmerkrankung erkrankten Hunden untersucht und mit derjenigen von Prednisolon verglichen. Als Endpunkt wurde die Verbesserung des „CIBD-Activity-Scores“ nach 6 Wochen herangezogen. Budesonid wurde in einer Dosierung von $0,2 \mathrm{mg} / \mathrm{kg}$ $1 \times$ tgl., Prednisolon in einer Dosierung von $1,0 \mathrm{mg} / \mathrm{kg} 2 \times \operatorname{tgl}$. eingesetzt. Die Dosis von Prednisolon wurde nach 3 Wochen halbiert. Es konnte kein statistisch signifikanter Unterschied in der Remission errechnet werden: 78\% bei Budesonid versus $69 \%$ bei Prednisolon. Auch konnte kein Unterschied in der Ausbildung unerwünschter Arzneimittelwirkungen festgestellt werden. Unter diesen Bedingungen besitzt Budesonid keinen therapeutischen Vorteil gegenüber Prednisolon [8].

Die genannten Studien liefern jedoch keinen Hinweis auf das optimale Dosierungsschema für Budesonid. Für eine abschließende Beurteilung der Wirksamkeit von Budesonid sowie von Kombinationen eines Glukokortikoids mit nicht immunsuppressiven Substanzen, z. B. Sucralfat, Sulfasalazin, Metronidazol, Tylosin, Ranitidin oder Omeprazol, stehen noch gut geplante prospektive Studien aus [3]. 


\section{Atopische Dermatitis des Hundes}

Die atopische Dermatitis des Hundes stellt eine der wenigen Erkrankungen beim Kleintier dar, zu der umfangreiche Konsensuspapiere zur klinischen Diagnostik und zur Wirksamkeit verschiedener Therapieoptionen existieren $[5,12]$. Sie zählt zu den allergisch-entzündlichen Erkrankungen, die mit Juckreiz und Hautveränderungen einhergeht. Als primärer Endpunkt zur Beurteilung der Wirksamkeit einer Therapie werden die Intensität des Juckreizes und der Umfang der Hautveränderungen anhand des „Canine Atopic Dermatitis Extent and Severity Index“ (CADESI) herangezogen. Sekundäre Endpunkte stellen die Remission der Symptome und die Ausbildung unerwünschter Arzneimittelwirkungen dar. Die Auswertung mehrerer aussagekräftiger, prospektiver randomisierter Studien ergab übereinstimmend eine gute Wirksamkeit von oral appliziertem Prednison, Prednisolon und Methylpred- nisolon mit einer Startdosis von $0,5 \mathrm{mg} /$ $\mathrm{kg}$ 1- oder 2-mal tgl. Eine vergleichbare Wirksamkeit konnte zudem für Hydrocortisonaceponat belegt werden, das als Spray $1 \times$ tgl. für 7 Tage lokal angewendet wird. Die Wirksamkeit der Glukokortikoide ist grundsätzlich mit derjenigen von Ciclosporin vergleichbar [12].

\section{Fazit}

Glukokortikoide sind hochwirksame Arzneimittel, die nicht wahllos verabreicht werden dürfen. Ihr Einsatz erfordert vielmehr eine strenge Indikationsstellung, die eine gründliche Diagnosestellung und umfassende Nutzen-RisikoAbwägung einschließt. Die klinische Wirksamkeit von Glukokortikoiden ist generell bei allen allergischen Erkrankungen, nicht infektiösen Entzündungen und Autoimmunerkrankungen gegeben. Ihr therapeutischer Nutzen ist ebenso bei einigen Schockformen und ausgewähl- ten Tumorerkrankungen belegt. Kontraindiziert sind Glukokortikoide dagegen aus heutiger Sicht beim akuten ZNSTrauma des Hundes. Bei der Diskopathie von Hund und Katze ist die Datenlage noch unvollständig, sodass keine allgemeine Empfehlung ausgesprochen werden kann.

Online zu finden unter

http://dx.doi.org/10.1055/s-0042-118780

\section{Literatur}

Die Literatur ist in der Online-Version unter www.Thieme-connect.de/products einsehbar.

\section{Prof. Dr. Hermann Ammer}

Fachtierarzt für Pharmakologie und Toxikologie Institut für Pharmakologie, Toxikologie und Pharmazie, Tierärztliche Fakultät München Königinstr. 16, 80539 München

h.ammer@Imu.de 


\section{Literatur}

1 Alderson P, Roberts I. Corticosteroids for acute traumatic brain injury. Cochrane Database Syst Rev 2005: 1

2 Behrend EN, Kemppainen RJ. Glucocorticoid therapy. Pharmacology, indications, and complications. Vet Clin North Am Small Anim Pract 1997; 27 (2): 187-213

3 Craven M, Simpson JW, Ridyard AE et al. Canine inflammatory disease: retrospective analysis of diagnosis and outcome in 80 cases (1995-2002). J Small Anim Pract 2004; 45 (7): 336-342

4 Crockcroft P, Holmes M. Handbook of Evidence-Based Veterinary Medicine. $5^{\text {th }}$ ed. Oxford: Blackwell Publishing; 2003

5 DeBoer DJ, Hillier A. The ACVD task force on canine atopic dermatitis (XV): fundamental concepts in clinical diagnosis. Vet Immunol Immunopathol 2001; 81 (3-4): 271-276

6 DiFazio J, Fletcher DJ. Updates in the management of the small animal patient with neurologic trauma. Vet Clin North Am Small Anim Pract 2013; 43 (4): 915-940

7 Dowling P. Corticosteroid and nonsteroidal antiinflammatory drug interactions. NAVC clinicians's Brief 2011; 89-92

8 Dye TL, Diehl KJ, Wheeler SL et al. Randomized, controlled trial of budesonide and prednisone for the treatment of idiopathic inflammatory bowel disease in dogs. J Vet Intern Med 2013; 27 (6): 1385-1391

9 Granger N, Smith PM, Jeffery ND. Clinical findings and treatment of non-infectious meningoencephalomyelitis in dogs: a systematic review of 457 published cases from 1962 to 2008. Vet J 2010; 184 (3): 290-297

10 Jergens AE, Crandell J, Morrison JA et al. Comparison of oral prednisone and prednisone combined with metronidazole for induction therapy of canine inflammatory bowel disease: a randomized-controlled trial. J Vet Intern Med 2010; 24 (2): 269-277

11 Narita T, Sato R, Motoishi K et al. The interaction between orally administered non-steroidal anti-inflammatory drugs and prednisolone in healthy dogs. J Vet Med Sci 2007; 69 (4): 353-363

12 Olivry T, Bizikova P. A systematic review of randomized controlled trials for prevention or treatment of atopic dermatitis in dogs: 2008-2011 update. Vet Dermatol 2013; 24 (1): 97-117
13 Park EH, White GA, Tieber LM. Mechanisms of injury and emergency care of acute spinal cord injury in dogs and cats. J Vet Emerg Crit Care (San Antonio) 2012; 22 (2): 160-178

14 Richter A, Ungemach FR. Pharmaka zur Beeinflussung von Entzündungen. In: Löscher W, Richter A, Potschka H, Hrsg. Pharmakotherapie bei Haus- und Nutztieren. 9. Aufl. Stuttgart: Enke; 2014: 446-486

15 Sande A, West C. Traumatic brain injury: a review of pathophysiology and management. J Vet Emerg Crit Care (San Antonio) 2010; 20 (2): 177-190 